\title{
Research Square \\ C2 Fracture Operative and Non-Operative Management Outcomes in Large Database Review
}

Christopher Foote ( $\nabla$ christopher.foote@uhsinc.com )

South Texas Health System - McAllen

Xuan-Lan Doan

Valley Health System

Cheryl Vanier

Touro University Nevada

Alejandro Betancourt

South Texas Health System - McAllen

Carlos Palacio

South Texas Health System - McAllen

\section{Research Article}

Keywords: Cervical spine fracture, odontoid fracture, trauma

Posted Date: February 18th, 2022

DOI: https://doi.org/10.21203/rs.3.rs-1229294/v2

License: (c) (1) This work is licensed under a Creative Commons Attribution 4.0 International License. Read Full License 


\section{Abstract}

\section{Background:}

Odontoid fractures are common and projected to increase. Comorbidities compound risk of surgery and balancing the risk of non-operative management is controversial. Stable fractures are managed based on patient comorbidity with suspected clinical prognosis. Type I and III fractures are typically managed safely with cervical orthoses. Management decisions of type II fractures however, come under frequent debate. This paper evaluates overall morbidity and mortality, and outcomes of operative and nonoperative management.

\section{Methods:}

We performed national database review of C2 fractures from January 2014 to December 2019. Patients were divided into categories based on Glasgow Coma Scale (GCS) and Injury Severity Score (ISS). Outcomes data considered hospital admission, Intensive Care Unit (ICU) admission, hospital length of stay (LOS), ICU LOS, and mortality. Logistic regression was used for mortality, hospital admission, and ICU admission. Odds ratios (OR) and 95\% confidence intervals (CI) were calculated from the logistic regression models. The Kruskal-Wallis test was used to compare the hospital and ICU LOS based on surgery overall, and by GCS and ISS.

\section{Results:}

42,003 patients were identified, 9,187 had surgery with overall mortality rate of $0.7 \%$. There was a younger operative median age (67) and interquartile range (IQR: 47,78$)$ than non-operative group (73, IQR: 56,83$)$. Both had the same median ISS score (10). Surgery was associated with lower rates of mortality, from $0.1 \%$ to $0.9 \%$ mortality for non-operative. Mild or moderate GCS mortality improved operative $(0.07 \%)$ to non-operative $(0.23 \%)$. Severe GCS patients with surgery had significantly improved mortality rates patients without ( $0.29 \%$ vs $7.69 \%$, respectively). Surgery increased ICU admissions for every ISS category. Severe GCS had higher chances of ICU admission, but no interaction with surgery. Operative patients had longer hospital and ICU stays. For all GCS and ISS categories, hospital and ICU LOS was longer for operative patients.

\section{Conclusions:}

This review demonstrates significant improvement in mortality with operative management. Standard non-operative management of type I and III C2 fractures is appropriate. Surgeons should consider operating on type II odontoid fractures unless patient cannot undertake the surgical risks of induction with general anesthesia.

Level of evidence: Level IV

\section{Background:}


Odontoid fractures are a common injury among the elderly [1] and with an ever-growing population, the number of injuries will continue. The frequency of injury and location of fracture on the dens of C2 vertebrae led Anderson and D'Alonzo to classify them as type I (fractures through the tip), type II (fractures through the base), and type III (fractures through the body of the C2 vertebral body) [2]. Consensus has established the validity of non-operative management with cervical collars for types I and III odontoid fractures, but the management of type II fractures continues to be a source for continued clinical debate. Odontoid fractures as a result of traumatic mechanism have a bimodal age distribution with peaks in younger adults aged 20-30, and elder adults aged 70-80 [3]. The younger group tend to incur injuries due to high velocity/high energy mechanism, while the elder group is thought to be injured during falls due to aging pathology $[3,4]$. It is this aging pathology that causes concern for optimal management as the anatomy of the fracture lends to poor healing due to weaker blood supply and poorer bone quality, as well as high risk for strain from transverse ligament $[4,5]$.

Due to comorbidities of the elderly and risk of surgery, as well as the risk of further injury in non-operative management, several papers have been published to try to glean evidence toward better decision-making. With the incidence of odontoid fractures reported as 21.4 per 100, 000 inpatient Medicare admissions and estimated costs over $\$ 1.5$ billion with the majority being elderly patients older than 65 [4], optimizing outcomes and improving patient care will decrease cost and further morbidity in an already vulnerable population. Unstable cervical fractures with high risk of compromising spinal cord are managed

operatively, but fractures with no immediate risk to spinal cord are managed based on clinical gestalt and balancing patient comorbidity with suspected clinical prognosis. Odontoid fracture mortality has been reported as $10-20 \%$ in 30-day, and $20-50 \%$ in 1 -year post-injury with low hemoglobin, admission from nursing facility, and neurological deficits (demonstrated by decreased GCS) as being predictors of mortality [4].

Non-operative management with cervical orthoses using a halo vest (HV), rigid cervical collar, or semirigid cervical collars (common brands: Philadelphia, Aspen, Miami J) are the usual management for stable type I and III odontoid fractures. Operative management is typically used in unstable odontoid and stable type II fractures, based on clinical judgement and weighing patient risk for surgery. The most common odontoid fracture is the type II being $65-74 \%$ of all reported odontoid fractures [6]. Management of stable type II odontoid fractures has come under frequent debate due to the unclear guidance or concrete clinical outcomes picture due to several patient factors and limited institutional studies. This paper aims to provide a large multicenter review of the American National Trauma Database system to evaluate overall morbidity and mortality in odontoid fractures, and the outcomes of operative and nonoperative management.

\section{Methods:}

The National Trauma Data Bank (NTDB) was searched for all C2 fractures from January 2014 to December 2019. Inclusion criteria consisted of International Classification of Diseases (ICD)-9 codes 805.02 or 805.12 and ICD-10 codes beginning with S12.1XX, as well as all ICD-9 and ICD-10 procedure 
codes included within the standard surgical procedures included in operative repair of $\mathrm{C} 2$ fractures. The patients were divided into categories based on Glasgow Coma Scale (GCS) of mild to moderate (GCS >8) and severe (GCS $\leq 8$ ). Injury Severity Score (ISS) was also categorized as minor (ISS 1-8), moderate (ISS 9-15), severe (ISS 16-24), and very severe (>24). Outcomes data that were considered included injury leading to hospital admission, injury leading to Intensive Care Unit (ICU) admission, hospital length of stay (LOS), ICU LOS, and mortality of injury. Patients were excluded for subjects who had died on index admission, or who were transferred to another hospital or left against medical advice. The ICU admission model included subjects who had been admitted to the hospital. Surgical intervention was the predictor variable of interest, and sex, age, race, ethnicity, ISS category, and GCS category were all considered as possible covariates or moderators between surgery and the outcome variables. A separate model was fit for each outcome variable. A logistic regression was used for mortality, hospital admission, and ICU admission.

The initial model included surgery, and a second model included surgery and all the possible covariates or moderators. A third model included the terms from the second model and the interactions between surgery and the possible covariates. Based on a log-likelihood test (alpha=0.05), interactions with evidence for statistical significance were followed up using descriptive statistics and estimating confidence intervals for proportions. This approach was taken because, even though the data set was large, the interactions produced some small categories which may have biased the estimates in the logistic regression. Odds ratios (OR) and the associated 95\% confidence intervals (Cl) were calculated from the first and second logistic regression models. For LOS outcomes, parametric models were a poor

fit, so the Kruskal-Wallis test was used to compare the hospital and ICU LOS for subjects who did and did not have surgery overall and by GCS and ISS. Analyses were done in Rv4.0.5.

\section{Results:}

A total of 42,003 subjects were included in the study, and 9,187 had surgery. Mortality rate was $0.7 \%$ (295 of 41,519; data for 484 was missing). The surgery group consisted of subjects who were slightly more likely to be male, were less likely to included Pacific Islanders and more likely to include American Indians and Hispanic or Latino, and who had relatively more individuals with severe or very severe ISS or severe GCS (Table 1). In both groups, the age range was 1 to 89 , but the surgery group subjects had younger median age (67) and interquartile range (IQR: 47,78 ) than for the non-surgery group (median: 73, IQR: 56, 83). Surgically managed and nonoperatively managed patients had the same median ISS score with surgery 10 (IQR: 9, 14) and nonoperative $10($ IQR: 9, 17). 
Table 1

Description of population based on surgical status.

\begin{tabular}{|c|c|c|c|c|}
\hline & $\mathbf{n}$ & Surgery & $\%$ surgery & pvalues \\
\hline Sex & & & & $<0.001$ \\
\hline Female & 21214 & 4199 & $20 \%$ & \\
\hline Male & 20789 & 4988 & $24 \%$ & \\
\hline Race & & & & $<0.001$ \\
\hline American.Indian & 179 & 55 & $31 \%$ & \\
\hline Asian & 514 & 120 & $23 \%$ & \\
\hline Black & 2731 & 759 & $28 \%$ & \\
\hline Pacific.Islander & 53 & 7 & $13 \%$ & \\
\hline Other & 1723 & 441 & $26 \%$ & \\
\hline White & 35833 & 7575 & $21 \%$ & \\
\hline ISS & & & & 0.009 \\
\hline Not Hispanic or Latino & 36128 & 7768 & $22 \%$ & \\
\hline Hispanic or Latino & 2110 & 505 & $24 \%$ & \\
\hline GCS & & & & 0.070 \\
\hline Mild to Moderate (>8; $96 \%$ were 15$)$ & 30547 & 2689 & $9 \%$ & \\
\hline Severe $(\leq 8 ; 78 \%$ were 3$)$ & 3380 & 691 & $20 \%$ & \\
\hline ISS & & & & $<0.001$ \\
\hline Minor & 6306 & 1385 & $22 \%$ & \\
\hline Moderate & 24687 & 5041 & $20 \%$ & \\
\hline Severe & 6897 & 1695 & $25 \%$ & \\
\hline Very Severe & 3629 & 913 & $25 \%$ & \\
\hline
\end{tabular}

\section{Mortality}

Even adjusted for covariates such as injury severity, age, and race, surgery was associated with substantially lower rates of mortality, from $0.1 \%$ mortality for subjects who had surgery to $0.9 \%$ mortality 
for subjects who did not have surgery (Table 2). The effect of surgery on mortality was moderated by the GCS (chi-squared $=10.7, p<0.001$ ). For subjects with mild or moderate GCS, mortality went from $0.07 \%$ with surgery to $0.23 \%$ with no surgery (Table 3 ). The effect was larger for subjects with severe GCS, where patients who had surgery had mortality rates that were more than an order of magnitude smaller than for severe GCS patients without surgery (mortality: $0.29 \%$ vs $7.69 \%$, respectively) (Table 3 and Figure 1 ). Mortality rates varied little by sex or race, and the ISS did not have a strong association with mortality. Subjects with a severe GCS had a higher mortality rate than subjects with mild or moderate scores. Mortality rates did not significantly vary by race (chi-squared $=6.0$, degrees of freedom $(d f)=5, p=0.311$ ). 
Table 2

Differences in mortality.

\begin{tabular}{|c|c|c|c|c|c|}
\hline & OR $(95 \% \mathrm{Cl})$ & p-value & Died & Lived & $\%$ mortality \\
\hline Surgery (Unadjusted) & $0.12(0.07,0.23)$ & $<0.001$ & & & \\
\hline \multicolumn{6}{|l|}{ Surgery (Adjusted) } \\
\hline No Surgery & & & 286 & 32199 & $0.9 \%$ \\
\hline With Surgery & $0.1(0.04,0.22)$ & $<0.001$ & 9 & 9025 & $0.1 \%$ \\
\hline \multicolumn{6}{|l|}{ Sex } \\
\hline Female & & & 127 & 20856 & $0.6 \%$ \\
\hline Male & $1.01(0.76,1.34)$ & 0.970 & 168 & 20364 & $0.8 \%$ \\
\hline Age (years) & $1.01(1,1.02)$ & 0.011 & $62(41,80)$ & $72(54,82)$ & \\
\hline \multicolumn{6}{|l|}{ ISS } \\
\hline Minor & & & 26 & 6280 & $0.4 \%$ \\
\hline Moderate & $0.49(0.28,0.87)$ & 0.015 & 60 & 24627 & $0.2 \%$ \\
\hline Severe & $0.78(0.43,1.44)$ & 0.434 & 56 & 6841 & $0.8 \%$ \\
\hline Very Severe & $1.54(0.86,2.78)$ & 0.148 & 153 & 3476 & $4.2 \%$ \\
\hline \multicolumn{6}{|l|}{ GCS } \\
\hline Severe & $21.9(14.8,32.4)$ & $<0.001$ & 206 & 3129 & $6.2 \%$ \\
\hline Mild or moderate & & & 68 & 35147 & $0.2 \%$ \\
\hline \multicolumn{6}{|l|}{ Race } \\
\hline White & & & 225 & 35273 & $0.6 \%$ \\
\hline American.Indian & $4.0(1.4,11.7)$ & 0.012 & 4 & 172 & $2.3 \%$ \\
\hline Asian & $1.2(0.4,3.4)$ & 0.702 & 4 & 489 & $0.8 \%$ \\
\hline Black & $1.1(0.7,1.7)$ & 0.681 & 28 & 2663 & $1.0 \%$ \\
\hline Pacific.Islander & $3.4(0.4,29.2)$ & 0.268 & 1 & 46 & $2.1 \%$ \\
\hline Other & $0.9(0.5,1.7)$ & 0.736 & 13 & 1613 & $0.8 \%$ \\
\hline \multicolumn{6}{|c|}{$\begin{array}{l}\text { Differences in mortality based on surgical intervention, sex, age, injury severity score (ISS), Glasgow } \\
\text { Coma Scale (GCS), and race. The odds ratio (OR) and } 95 \% \text { confidence interval (CI), the p-value based } \\
\text { on logistic regression, and the number of subjects with a characteristic who died and lived, as well the } \\
\text { percent mortality for the group, are reported. For age, median and inter-quartile range are reported for } \\
\text { each group. }\end{array}$} \\
\hline
\end{tabular}


Table 3

Mortality with and without surgery by GCS category.

\begin{tabular}{|c|c|c|c|c|c|c|}
\hline GCS & Surgery & Died & Lived & Mortality & $95 \% \mathrm{Cl}$ & \\
\hline \multirow[t]{2}{*}{ GCS Mild or Moderate } & No Surgery & 63 & 27521 & $0.23 \%$ & $0.18 \%$ & $0.29 \%$ \\
\hline & With Surgery & 5 & 7626 & $0.07 \%$ & $0.02 \%$ & $0.16 \%$ \\
\hline \multirow[t]{2}{*}{ GCS Severe } & No Surgery & 204 & 2448 & $7.69 \%$ & $6.72 \%$ & $8.79 \%$ \\
\hline & With Surgery & 2 & 681 & $0.29 \%$ & $0.05 \%$ & $1.17 \%$ \\
\hline
\end{tabular}

\section{Rates of Admission to Hospital or ICU}

The rate of admission to the hospital was $97.1 \%$. Surgery increased the admission rate from $96.8-98.5 \%$ (Table 4), but outcomes varied by ISS (chi-squared=20.7, df=3, $\mathrm{p}<0.001$ ). For subjects with minor or moderate ISS, surgery slightly increased the chances of hospital admission for those with minor or moderate ISS, whereas surgery did not change the chances of hospitalization for those with severe or very severe ISS (Table 5). The rate of admission to the ICU for those admitted to the hospital was $49.8 \%$, and surgery increased the admission rate to the ICU from $46.0 \%$ for those without surgery to $62.8 \%$ for those with surgery (Table 6). As with hospital admissions, likelihood of admission varied by ISS (chisquared $=10.4, d f=3, p=0.015)$. Surgery increased ICU admissions for every ISS category (Table 6, 7). Subjects with severe GCS had higher chances of ICU admission, but there was no interaction with surgery (chi-squared=0.9, $d f=1, p=0.470$ ). 
Table 4

Differences in hospitalization

\begin{tabular}{|c|c|c|c|c|c|}
\hline & OR (95\% Cl) & p-value & Admitted & Not admitted & $\%$ Admitted \\
\hline Surgery (Unadjusted) & $2.16(1.79,2.59)$ & $<0.001$ & & & \\
\hline \multicolumn{6}{|l|}{ Surgery (Adjusted) } \\
\hline No Surgery & & & 28447 & 953 & $96.8 \%$ \\
\hline With Surgery & $2.46(2,3.03$ & $<0.001$ & 8370 & 130 & $98.5 \%$ \\
\hline \multicolumn{6}{|l|}{ Sex } \\
\hline Female & & & 18661 & 523 & $97.3 \%$ \\
\hline Male & $0.92(0.8,1.06)$ & 0.257 & 18152 & 560 & $97.0 \%$ \\
\hline Age (years) & $1.01(1.01,1.10)$ & $<0.001$ & $72(54,82)$ & $69(49,80)$ & \\
\hline \multicolumn{6}{|l|}{ ISS } \\
\hline Minor & & & 5349 & 292 & $94.8 \%$ \\
\hline Moderate & $1.6(1.37,1.88)$ & $<0.001$ & 21810 & 703 & $96.9 \%$ \\
\hline Severe & $5.26(3.91,7.08)$ & $<0.001$ & 6402 & 64 & $99.0 \%$ \\
\hline Very Severe & $5.37(3.44,8.4)$ & $<0.001$ & 3256 & 24 & $99.3 \%$ \\
\hline \multicolumn{6}{|l|}{ GCS } \\
\hline Mild or moderate & & & 31424 & 988 & $97.0 \%$ \\
\hline Severe & $2.5(1.6,3.9)$ & $<0.001$ & 2948 & 23 & $99.2 \%$ \\
\hline \multicolumn{6}{|l|}{ Race } \\
\hline White & & & 31470 & 908 & $97.2 \%$ \\
\hline American.Indian & $1.4(0.4,4.3)$ & 0.597 & 153 & 3 & $98.1 \%$ \\
\hline Asian & $1.0(0.6,1.9)$ & 0.955 & 449 & 12 & $97.4 \%$ \\
\hline Black & $0.7(0.6,0.9)$ & 0.004 & 2373 & 104 & $95.8 \%$ \\
\hline Pacific.Islander & $1.2(0.2,9.2)$ & 0.832 & 42 & 1 & $97.7 \%$ \\
\hline Other & $1.1(0.8,1.6)$ & 0.499 & 1477 & 35 & $97.7 \%$ \\
\hline \multicolumn{6}{|c|}{$\begin{array}{l}\text { Hospitalization rate based on surgical intervention, sex, age, injury severity score (ISS), Glasgow } \\
\text { Coma Scale (GCS), and race. The odds ratio and } 95 \% \text { confidence interval (CI), the p-value based on } \\
\text { logistic regression, and the number of subjects with a characteristic who were and were not admitted } \\
\text { as well the admission rate for the group, are reported. For age, median and inter-quartile range are } \\
\text { reported for each group. }\end{array}$} \\
\hline
\end{tabular}


Table 5

Effect of GCS on hospital admission with and without surgery.

\begin{tabular}{|c|c|c|c|c|c|}
\hline ISS & Surgery & Admitted & Not admitted & Hospital admission & $95 \% \mathrm{Cl}$ \\
\hline \multirow[t]{2}{*}{ Minor } & No Surgery & 4105 & 252 & $94.22 \%$ & $(93.47,94.88)$ \\
\hline & With Surgery & 1244 & 40 & $96.88 \%$ & $(95.74,97.74)$ \\
\hline \multirow[t]{2}{*}{ Moderate } & No Surgery & 17165 & 639 & $96.41 \%$ & $(96.12,96.68)$ \\
\hline & With Surgery & 4645 & 64 & $98.64 \%$ & $(98.25,98.94)$ \\
\hline \multirow[t]{2}{*}{ Severe } & No Surgery & 4790 & 47 & $99.03 \%$ & $(98.69,99.28)$ \\
\hline & With Surgery & 1612 & 17 & $98.96 \%$ & $(98.29,99.37)$ \\
\hline \multirow[t]{2}{*}{ Very Severe } & No Surgery & 2387 & 15 & $99.38 \%$ & $(98.94,99.64)$ \\
\hline & With Surgery & 869 & 9 & $98.97 \%$ & $(97.98,99.50)$ \\
\hline
\end{tabular}


Table 6

Differences in ICU admission

\begin{tabular}{|c|c|c|c|c|c|}
\hline & OR $(95 \% \mathrm{Cl})$ & p-value & Admitted & Not admitted & $\%$ Admitted \\
\hline Surgery (Unadjusted) & $1.98(1.88,2.08)$ & $<0.001$ & & & \\
\hline \multicolumn{6}{|l|}{ Surgery (Adjusted) } \\
\hline No Surgery & & & 13129 & 15394 & $46.0 \%$ \\
\hline With Surgery & $2.02(1.9,2.14)$ & $<0.001$ & 5267 & 3123 & $62.8 \%$ \\
\hline \multicolumn{6}{|l|}{ Sex } \\
\hline Female & & & 8446 & 10268 & $45.1 \%$ \\
\hline Male & $1.21(1.15,1.27)$ & $<0.001$ & 9946 & 8249 & $54.7 \%$ \\
\hline Age (years) & $1(1,1)$ & 0.666 & $69(50,81)$ & $75(59,83)$ & \\
\hline \multicolumn{6}{|l|}{ ISS } \\
\hline Minor & & & 1800 & 3569 & $33.5 \%$ \\
\hline Moderate & $1.47(1.36,1.58)$ & $<0.001$ & 9023 & 12850 & $41.3 \%$ \\
\hline Severe & $4.43(4.05,4.84)$ & $<0.001$ & 4543 & 1866 & $70.9 \%$ \\
\hline Very Severe & $14.33(12.17,16.91)$ & $<0.001$ & 3030 & 232 & $92.9 \%$ \\
\hline \multicolumn{6}{|l|}{ GCS } \\
\hline Mild or Moderate & $15.6(12.5,19.5)$ & $<0.001$ & 14566 & 16946 & $46.2 \%$ \\
\hline Severe & $1(1,1)$ & $<0.001$ & 2846 & 105 & $96.4 \%$ \\
\hline \multicolumn{6}{|l|}{ Race } \\
\hline White & & & 15264 & 16286 & $48.4 \%$ \\
\hline American.Indian & $1.1(0.7,1.6)$ & 0.654 & 92 & 62 & $59.7 \%$ \\
\hline Asian & $1.1(0.9,1.4)$ & 0.498 & 239 & 212 & $53.0 \%$ \\
\hline Black & $1.1(1,1.2)$ & 0.136 & 1348 & 1032 & $56.6 \%$ \\
\hline Pacific.Islander & $1.5(0.7,3.3)$ & 0.285 & 26 & 16 & $61.9 \%$ \\
\hline Other & $1.3(1.2,1.5)$ & $<0.001$ & 921 & 558 & $62.3 \%$ \\
\hline \multicolumn{6}{|c|}{$\begin{array}{l}\text { ICU admission rate based on surgical intervention, sex, age, injury severity score (ISS), Glasgow Coma } \\
\text { Scale (GCS), and race. The odds ratio and } 95 \% \text { confidence interval (CI), the p-value based on logistic } \\
\text { regression, and the number of subjects with a characteristic who were and were not admitted, as well } \\
\text { the admission rate for the group, are reported. For age, median and inter-quartile range are reported } \\
\text { for each group. }\end{array}$} \\
\hline
\end{tabular}


Table 7

ICU admission rates with and without surgery by ISS category.

\begin{tabular}{|llllll|}
\hline ISS & Surgery & Admitted & Not admitted & Hospital admission & 95\% Cl \\
\hline Minor & No Surgery & 1274 & 2847 & $30.91 \%$ & $(29.51,32.36)$ \\
\hline \multirow{2}{*}{ Moderate } & With Surgery & 526 & 722 & $42.15 \%$ & $(39.39,44.95)$ \\
\hline \multirow{2}{*}{ Severe } & No Surgery & 6429 & 10787 & $37.34 \%$ & $(36.62,38.07)$ \\
\hline & With Surgery & 2594 & 2063 & $55.70 \%$ & $(54.25,57.13)$ \\
\hline Very Severe & No Surgery & 3228 & 1567 & $67.32 \%$ & $(65.96,68.64)$ \\
\hline \multicolumn{7}{|l}{$\begin{array}{l}\text { The number of subjects who died and lived and mortality with } 95 \% \text { confidence interval } \\
\text { reported for each combination of surgery and injury severity score (ISS). }\end{array}$} \\
\hline
\end{tabular}

\section{LOS in Hospital and ICU}

Overall, subjects who had surgery had longer hospital and ICU stays than subjects who did not have surgery (Table 8, 9). For all GCS and ISS categories, the difference in hospital LOS and ICU between surgery and no surgery groups was larger for categories indicating more severely injured subjects. For example, subjects with mild or moderate GCS stayed a median of five days without surgery and seven days with surgery. For subjects with severe GCS and no surgery, LOS was a median of six days, whereas it was 17 days with surgery (Table 8). 
Table 8

Hospital length of stay (LOS) with and without surgery by GCS and ISS categories.

\begin{tabular}{|c|c|c|c|c|c|c|}
\hline & & Surgery & $\mathbf{n}$ & LOS & W & p-value \\
\hline \multicolumn{2}{|c|}{ All Subjects } & No Surgery & 28104 & $5(3,8)$ & 2386 & $<0.001$ \\
\hline & & With Surgery & 8349 & $7(5,8)$ & & \\
\hline \multirow[t]{4}{*}{ GCS } & Mild or Moderate & No Surgery & 24060 & $5(3,7)$ & 1995 & $<0.001$ \\
\hline & & With Surgery & 7092 & $7(5,12)$ & & \\
\hline & Severe & No Surgery & 2222 & $6(2,17)$ & 268 & $<0.001$ \\
\hline & & With Surgery & 655 & $17(9,28)$ & & \\
\hline \multirow[t]{3}{*}{ ISS } & Minor & No Surgery & 4044 & $3(2,5)$ & 243 & $<0.001$ \\
\hline & & With Surgery & 1242 & $5(3,8)$ & & \\
\hline & \multirow[t]{2}{*}{ Moderate } & No Surgery & 17001 & $4(3,7)$ & 1545 & $<0.001$ \\
\hline & & With Surgery & 4633 & $7(4,10)$ & & \\
\hline \multirow{2}{*}{\multicolumn{2}{|c|}{ Severe }} & No Surgery & 4728 & $7(4,11)$ & 340 & $<0.001$ \\
\hline & & With Surgery & 1609 & $10(6,17)$ & & \\
\hline \multirow{2}{*}{\multicolumn{2}{|c|}{ Very Severe }} & No Surgery & 2331 & $8(3,18)$ & 313 & $<0.001$ \\
\hline & & With Surgery & 865 & $17(10,28)$ & & \\
\hline
\end{tabular}


Table 9

ICU length of stay (LOS) with and without surgery by GCS and ISS categories.

\begin{tabular}{|c|c|c|c|c|c|c|}
\hline & & Surgery & $\mathbf{n}$ & ICU LOS & W & p-value \\
\hline \multirow[t]{2}{*}{ All Subjects } & & No Surgery & 11763 & $3(2,6)$ & 404 & $<0.001$ \\
\hline & & With Surgery & 3449 & $5(3,11)$ & & \\
\hline \multirow[t]{4}{*}{ GCS } & Mild or Moderate & No Surgery & 9708 & $3(2,5)$ & 312 & $<0.001$ \\
\hline & & With Surgery & 2846 & $5(3,9)$ & & \\
\hline & Severe & No Surgery & 1349 & $5(2,12)$ & 158 & $<0.001$ \\
\hline & & With Surgery & 372 & $13(7,21)$ & & \\
\hline \multirow[t]{8}{*}{ ISS } & Minor & No Surgery & 1230 & $2(2,3)$ & 26 & $<0.001$ \\
\hline & & With Surgery & 340 & $3(2,4)$ & & \\
\hline & Moderate & No Surgery & 6293 & $3(2,4)$ & 178 & $<0.001$ \\
\hline & & With Surgery & 1625 & $4(2,7)$ & & \\
\hline & Severe & No Surgery & 2630 & $4(2,7)$ & 100 & $<0.001$ \\
\hline & & With Surgery & 902 & $6(3,12)$ & & \\
\hline & Very Severe & No Surgery & 1610 & $5(2,13)$ & 160 & $<0.001$ \\
\hline & & With Surgery & 582 & $12(6,20)$ & & \\
\hline
\end{tabular}

\section{Discussion:}

In the United States NTDB data between January 2014 to December 2019, overall mortality rate in all odontoid fractures was low at $0.7 \%$, with lower mortality in those undergoing operative management in either the mild-moderate and severe GCS groups. Outpatient follow-up and care is not tracked through the NTDB, but longer-term odontoid fracture mortality has been reported as $10-20 \%$ in 30 -day, and $20-50 \%$ in 1-year post-injury with most mortalities related to patient comorbidities and not sequelae from odontoid fracture injuries [4]. Therefore, the immediate mortality of the odontoid fracture is associated with the index admission and management therein. The data demonstrates that mortality is improved with operative management, having a mortality rate of $0.1 \%$ compared to non-operative management mortality rate at $0.9 \%$. ISS scores between the two groups were very similar, but GCS was found to be a significant indicator of mortality in this study with OR of $21.9(14.8,32.495 \% \mathrm{Cl})$ for severe GCS compared with mild to moderate GCS. Severe GCS in traumatic injuries is likely due to acute brain injury and not a chronic comorbidity of pre-existing conditions for determining pre-operative suitability for 
which the Charlson Comorbidity Index (CCl) [10] or the American College of Surgeons National Surgical Quality Improvement Program (ACS NSQIP) [11] were designed to aid in clinical decision making. Decisions on whether to operate then become based on weighing the clinical stability of the patient in front of the surgeon and the risk of the non-operative and surgical intervention options.

Non-operative management techniques have been presented as viable options to mitigate patient complications with studies providing evidence of fracture fusion in selected patients. These selected patients are typically of lower severity (lower ISS) and more mild-moderate GCS score. Patients that are deemed too high risk for surgery are also managed non-operatively as the patient comorbidity, frailty, or other perioperative risks lend greater risk to the patient than the injury itself. In these cases, cervical immobilization using one of the methods listed above are utilized. Historically, the halo vest was used for maintaining cervical in-line stabilization for healing but require precise pin placement and have risks of infection, nerve/brain damage, and provide the patient with pain and tissue breakdown beneath the torso, and functional limitation and poor compliance $[4,6-8]$. Semi-rigid collars were shown to have fewer complications, with similar or improved fracture union ( $>70 \%$ semi-rigid vs $40 \% \mathrm{HV})[4,6,8,9]$. Differing opinions exist to whether only full osseous fusion or including asymptomatic pseudoarthrosis are considered sufficient in goals of care with these fractures. Measures of quality of life, pain, and neck mobility are taken into consideration for cessation of non-operative management with cervical orthosis, with some still requiring surgery for failure of non-operative management. Stable nonunion or asymptomatic mobile nonunion have been deemed appropriate for clinical outcome in geriatric patients whose operative morbidity and mortality associated with undergoing surgery [4].

Patient comorbidities in odontoid fractures can be misleading as deciding factors for surgery as occult injuries can be missed on radiological studies alone. Stability of injury by measuring anteroposterior displacement $>3.5 \mathrm{~mm}$ and perceived spinal canal invasion are insufficient for injury severity. Agunbiade, et al. described a patient with complete cervical cord transection from a ground-level fall in a patient with minimal radiological evidence for spinal cord injury from mildly displaced odontoid fracture [12]. Disruption of the ligamentous tissues and re-alignment in cervical collar during emergency evaluation with routine imaging could miss neurological injury. Suspicion is usually low of severe spinal cord injury as it is typically rare and patients may be intubated, but this must always be considered in seriously sick patients. When taken for MRI, ligamentous injuries may then be found with even minor fractures. Ligamentous injuries may still cause significant comorbidity themselves as sub-failure injury to soft tissues can still cause persistent pain, headaches, dizziness, and chronic spine pain [13].

Cervical ligaments placed under stress during the traumatic injury but not rupturing can still create structural damage causing spinal instability and chronic pain leading to increased future falls or injury. Therefore, even when odontoid fractures are managed non-operatively with fracture union, patients may be left with persistent cervical instability [13]. Lending to cervical instability in these patients is a study by Kepler, et al. that demonstrated $30 \%$ of acute traumatic injury with initial findings of type II odontoid fractures had chronic $\mathrm{C} 2$ injuries with obvious nonunion from poor primary healing, with $17.5 \%$ of these showing new neurologic deficits on secondary injury [14]. These patients in that study were new to the 
hospital where this study was performed and did not state whether the patient had known of prior diagnosis of odontoid fracture or treatment non-operatively. The number of chronic type Il odontoid fracture patients and their likelihood of returning to the emergency department for subsequent injuries found in this single institution may demonstrate an underrepresentation of morbidity for non-operative management causing increased evidence for early operative management.

Operative management techniques for odontoid fractures are divided between anterior and posterior approaches. Anterior cannulated screw fixation (ACSF) is the gold-standard for odontoid fracture surgery and has shown union rates of $88-100 \%[15,16]$. The operation requires anterior exposure through the prevertebral fascia to place screw through the body of $\mathrm{C} 2$ into the dens fragment to pull it towards the fracture line for fusion. This approach provides immediate stabilization, reduced post-operative pain, no bone grafting, and preservation of the atlanto-axial rotational movement [15]. The neck disability index (NDI) measuring the subjective pain and discomfort of the patient was rated as better than posterior instrumentation of C1-2 without fusion (PIWF) but showed decreased union rates (90.9\% ACSF vs 96\% PIWF; not statistically significant in that study) [15]. Both approaches demonstrate much higher successful union rates compared to non-operative management $(90.9-96 \%$ vs $40-70 \%)[4,6-9,14,15]$. PIWF involves a second operation to remove the instrumentation, so the patient is put at risk for surgical complications again in order to return some of the axial rotation of the cervical spine, but still not as much as present in ACSF. Another posterior approach is a posterior instrumentation with fusion (PIF) where bone graft is used to fuse the C1-C2 vertebrae to achieve immediate cervical stability but a loss of approximately $50 \%$ of axial rotation [4], however it requires only a single operation with $90.5 \%$ union rate compared to $96 \%$ union rate in PIWF [17]. This approach reduces functionality but saves the patient from additional surgery if patient comorbidity and risk of reoperation is a deciding factor. Another approach is an occipito-cervical fusion (OCF) which is typically used when a posterior decompression is needed. This significantly reduces in cervical mobility, increases risk of dysphagia, has lower fusion rates, and significant post-operative complications as the procedure requires instrumentation along the occiput through $\mathrm{C} 1$ and $\mathrm{C} 2$ [18].

Comparing all odontoid fractures in this large national database, low mortality in operative management for all types has shown better outcomes than non-operative management. Given that the standard management of type I and type III odontoid fractures are nonoperative with cervical collars, the operative patients are likely the unstable fractures and type II odontoid fractures. Patients undergoing surgery have demonstrated longer hospital length of stay and increased likelihood of ICU admission. This may represent the time required for post-operative recovery as an operation was associated with longer ICU and hospital LOS for each level of ISS and GCS groups. Longer hospital and ICU LOS in severe GCS compared to mild to moderate GCS, and similarly with more severe ISS scores, likely is associated to other injuries as operative fixation of the fracture immediately stabilizes the odontoid injury. Wagner, et al. reported that non-operative management of type II odontoid fractures led to hazard ratios for mortality at follow-up was 1.35 and an eventual delayed operation of $22 \%$ of patients [19]. 
In the elderly population incurring these injuries, the bone quality and blood supply necessary for nonoperative fracture healing was a comorbid risk to optimal healing [19]. Physiology and pre-existing comorbid conditions might be indications toward operative management due to chronic pain and complications leading to eventual and costly continued management. Surgery was not found to have a negative impact on survival with adjusted comorbidities in the AOSpine North America study by Chapman, et al [20]. Mitigating cost and risk associated with prolonged non-operative management in elderly patients 65-84 would be more beneficial to undergo an operation when considering cost at $\$ 100,000$ /quality-adjusted life year (QALY) as a surgical benchmark [19]. The longer hospital and ICU LOS associated with surgical healing and management of acute injuries may be marginal financial cost by operating on type II odontoid fractures relative to the opportunity cost of prolonged outpatient clinic hours, subsequent operative time to the surgeon and chronic pain, medication effects, and quality of life lost by patients.

\section{Conclusions:}

Odontoid fractures continue to be a common injury and despite multiple publications discussing management, especially with the focus being type II odontoid fractures, there does not appear to be a large enough review to define an optimal decision. Several literature reviews have summarized individual institutional data and described various surgical techniques to manage these fractures with evidence that good long-term clinical outcomes can be achieved from operative management over non-operative management. There have been several surgical approaches described that provide options to the surgeon for handling acceptable post-operative complications while fixing the fracture depending on the frailty or clinical status of the pre-operative patient. The data analyzed in this study of a large national database review demonstrates that statistically significant improvement in mortality can be achieved with operative management in the acute setting with adjusted patient demographics and comorbidities. Injury severity appears to be similar in both operative and non-operative management cohorts to reliably compare the two based on surgical intervention. Overall patient care with acceptance of marginal increase in financial costs due to hospital and ICU stays associated with surgical intervention are preferable to opportunity cost of prolonged management of patients and complications associated with no intervention. Based on these data, unless the patient cannot undergo the surgical risks of induction with general anesthesia, we recommend be managed operatively with one of the approaches described rather than under non-operative management.

\section{Abbreviations}

ACS NSQIP

American College of Surgeons National Surgical Quality Improvement Program ACSF

anterior cannulated screw fixation

$\mathrm{CCl}$ 
Charlson Comorbidity Index

$\mathrm{Cl}$

confidence interval

C2

Cervical vertebrae 2; odontoid

df

degrees of freedom

GCS

Glasgow Coma Scale

HV

halo vest

ICD

International Classification of Diseases

ICU

intensive care unit

IQR

interquartile range

ISS

Injury Severity Score

LOS

length of stay

NDI

neck disability index

NTDB

National Trauma Database

OCF

occipito-cervical fusion

OR

odds ratio

PIF

posterior instrumentation with fusion

PIWF

posterior instrumentation without fusion

QALY

quality-adjusted life year

\section{Declarations}

\section{Ethics approval and consent to participate}

National Trauma Database review; not applicable. 


\section{Consent for publication}

Not applicable

\section{Availability of data and material}

The datasets generated and/or analyzed during the current study are available via the American College of Surgeons National Trauma Data Bank as described in Methods.

\section{Competing interests}

We know of no conflicts of interests associated with the publication and there has been no financial support for this work that could influence its outcome.

\section{Funding}

There was no outside financial support for the production of this article.

\section{Authors' contributions}

CF and XD completed data collection. CF and CHP wrote the manuscript. CV performed statistical analysis. $A B$ was senior mentor for this paper. The author(s) read and approved the final manuscript.

\section{Acknowledgements}

Not applicable.

\section{Author Details}

${ }^{1}$ South Texas Health System Trauma Department, McAllen Medical Center, McAllen Texas; ${ }^{2}$ Valley Health System General Surgery Residency Program, Las Vegas Nevada; ${ }^{3}$ Touro University Nevada College of Osteopathic Medicine, Touro University Nevada

\section{References}

1. Ryan MD, Henderson JJ. The epidemiology of fractures and fracture-dislocations of the cervical spine. Injury. 1992;23(1):38-40. doi: 10.1016/0020-1383(92)90123-a. PMID: 1541497.

2. Anderson LD, D'Alonzo RT. Fractures of the odontoid process of the axis. J Bone Joint Surg Am. 1974 Dec;56(8):1663-74. PMID: 4434035.

3. Robinson AL, Möller A, Robinson Y, Olerud C. C2 Fracture Subtypes, Incidence, and Treatment Allocation Change with Age: A Retrospective Cohort Study of 233 Consecutive Cases. Biomed Res Int. 2017;2017:8321680. doi: 10.1155/2017/8321680. Epub 2017 Jan 15. PMID: 28182084; PMCID: PMC5274671. 
4. lyer S, Hurlbert RJ, Albert TJ. Management of Odontoid Fractures in the Elderly: A Review of the Literature and an Evidence-Based Treatment Algorithm. Neurosurgery. 2018 Apr 1;82(4):419-430. doi: 10.1093/neuros/nyx546. PMID: 29165688.

5. Chapman J, Smith JS, Kopjar B, Vaccaro AR, Arnold P, Shaffrey Cl, Fehlings MG. The AOSpine North America Geriatric Odontoid Fracture Mortality Study: a retrospective review of mortality outcomes for operative versus nonoperative treatment of 322 patients with long-term follow-up. Spine (Phila Pa 1976). 2013 Jun 1;38(13):1098-104. doi: 10.1097/BRS.0b013e318286f0cf. PMID: 23354104; PMCID: PMC3678887.

6. Gembruch O, Lemonas E, Ahmadipour Y, Sure U, El Hindy N, Müller O. Nonoperative management of C-2 dens fractures: Single center experience and review of the literature. Clin Neurol Neurosurg. 2018 Jun;169:166-173. doi: 10.1016/j.clineuro.2018.04.021. Epub 2018 Apr 22. PMID: 29705652.

7. Müller EJ, Schwinnen I, Fischer K, Wick M, Muhr G. Non-rigid immobilisation of odontoid fractures. Eur Spine J. 2003 Oct;12(5):522-5. doi: 10.1007/s00586-003-0531-1. Epub 2003 May 14. PMID: 12748895; PMCID: PMC3468001.

8. Girardo M, Rava A, Gargiulo G, Coniglio A, Artiaco S, Massè A, Fusini F. Clinical and radiological union rate evaluation of type 2 odontoid fractures: A comparison between anterior screw fixation and halo vest in elderly patients. J Craniovertebr Junction Spine. 2018 Oct-Dec;9(4):254-259. doi: 10.4103/jcvjs.JCVJS_93_18. PMID: 30783350; PMCID: PMC6364356.

9. Aquila F, Tacconi L, Baldo S. Type II Fractures in Older Adults: Can They Be Treated Conservatively?: A Single-Center Experience and Review of the Literature. World Neurosurg. 2018 Oct;118:e938-e945. doi: 10.1016/j.wneu.2018.07.105. Epub 2018 Jul 20. PMID: 30036717.

10. Glasheen WP, Cordier T, Gumpina R, Haugh G, Davis J, Renda A. Charlson Comorbidity Index: ICD-9 Update and ICD-10 Translation. Am Health Drug Benefits. 2019 Jun-Jul;12(4):188-197. PMID: 31428236; PMCID: PMC6684052.

11. American College of Surgery National Surgical Quality Improvement Program. https://riskcalculator.facs.org/RiskCalculator/index.jsp. Last accessed 12/27/2021.

12. Agunbiade S, Belton PJ, Mesfin FB. Spinal Cord Transection in a Type II Odontoid Fracture From a Ground-Level Fall. Cureus. 2020 Dec 28;12(12):e12342. doi: 10.7759/cureus.12342. PMID: 33520537; PMCID: PMC7837629.

13. Trajkovski A, Hribernik M, Kunc R, Kranjec M, Krašna S. Analysis of the mechanical response of damaged human cervical spine ligaments. Clin Biomech (Bristol, Avon). 2020 May;75:105012. doi: 10.1016/j.clinbiomech.2020.105012. Epub 2020 Apr 19. PMID: 32371284.

14. Kepler CK, Vaccaro AR, Dibra F, Anderson DG, Rihn JA, Hilibrand AS, Harrop JS, Albert TJ, Radcliff KE. Neurologic injury because of trauma after type II odontoid nonunion. Spine J. 2014 Jun 1;14(6):9038. doi: 10.1016/j.spinee.2013.07.443. Epub 2013 Sep 27. PMID: 24080191.

15. Yuan S, Wei B, Tian Y, Yan J, Xu W, Wang L, Liu X. The comparison of clinical outcome of fresh type II odontoid fracture treatment between anterior cannulated screws fixation and posterior 
instrumentation of C1-2 without fusion: a retrospective cohort study. J Orthop Surg Res. 2018 Jan 8;13(1):3. doi: 10.1186/s13018-017-0702-0. PMID: 29310670; PMCID: PMC5759802.

16. Yuan S, Wei B, Tian Y, Yan J, Xu W, Wang L, Liu X. Posterior temporary C1-2 fixation for 3-part fractures of the axis (odontoid dens and Hangman fractures). Medicine (Baltimore). 2018 Nov;97(48):e12957. doi: 10.1097/MD.0000000000012957. PMID: 30508884; PMCID: PMC6283111.

17. Guo Q, Deng Y, Wang J, Wang L, Lu X, Guo X, Ni B. Comparison of Clinical Outcomes of Posterior C1C2 Temporary Fixation Without Fusion and C1-C2 Fusion for Fresh Odontoid Fractures. Neurosurgery. 2016 Jan;78(1):77-83. doi: 10.1227/NEU.0000000000001006. PMID: 26348006.

18. Bhimani AD, Chiu RG, Esfahani DR, Patel AS, Denyer S, Hobbs JG, Mehta Al. C1-C2 Fusion Versus Occipito-Cervical Fusion for High Cervical Fractures: A Multi-Institutional Database Analysis and Review of the Literature. World Neurosurg. 2018 Nov;119:e459-e466. doi:

10.1016/j.wneu.2018.07.182. Epub 2018 Jul 30. PMID: 30071333.

19. Wagner SC, Schroeder GD, Kepler CK, Schupper AJ, Kandziora F, Vialle EN, Oner C, Fehlings MG, Vaccaro AR. Controversies in the Management of Geriatric Odontoid Fractures. J Orthop Trauma. 2017 Sep;31 Suppl 4:S44-S48. doi: 10.1097/BOT.0000000000000948. PMID: 28816875.

\section{Figures}

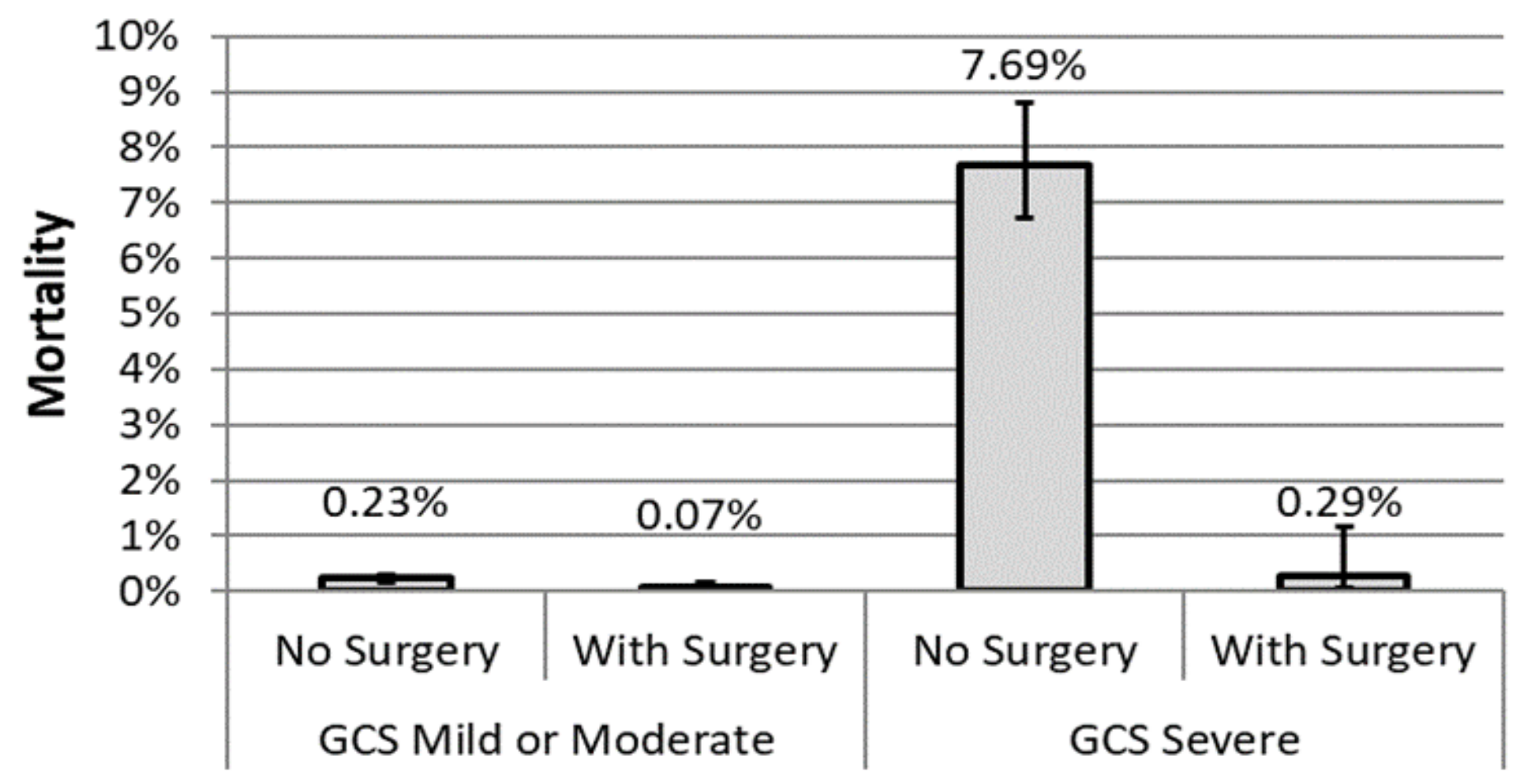

Figure 1

Mortality with and without surgery by GCS category. 